\title{
OPEN Mode Selective Up-conversion Detection with Turbulence
}

\begin{abstract}
He Zhang ${ }^{1,2}$, Santosh Kumar ${ }^{1,2}$ \& Yu-Ping Huang ${ }^{1,2^{*}}$
We experimentally study a nonlinear optical approach to selective manipulation and detection of structured images mixed with turbulent noise. Unlike any existing adaptive-optics method by applying compensating modulation directly on the images, here we account for the turbulence indirectly, by modulating only the pump driving the nonlinear process but not the images themselves. This indirect approach eliminates any signal modulation loss or noise, while allowing more flexible and capable operations. Using specifically sum frequency generation in a lithium niobate crystal, we demonstrate selective upconversion of Laguerre-Gaussian spatial modes mixed with turbulent noise. The extinction reaches $\sim 40 \mathrm{~dB}$ without turbulence, and maintains $\sim 20 \mathrm{~dB}$ in the presence of strong turbulence. This technique could find utilities in classical and quantum communications, compressive imaging, pattern recognition, and so on.
\end{abstract}

Nonlinear optics (NLO) underpins optical parametric oscillation ${ }^{1,2}$, parametric downconversion ${ }^{3,4}$, harmonic generation $^{5,6}$, sum-frequency generation ${ }^{7,8}$, four wave mixing ${ }^{9,10}$, etc., with wide applications in optical communications ${ }^{11,12}$, biomedical engineering ${ }^{13}$, metrology ${ }^{14}$, and quantum information ${ }^{15}$. For optical signal processing and detection, NLO techniques can offer significant advantages over their linear-optics counterparts ${ }^{5,16,17}$, as demonstrated repeatedly in temporal mode-selective frequency conversion ${ }^{18-24}$, lossless photon shaping ${ }^{25}$, spiral phase contrast imaging of the edges ${ }^{26}$, and field-of-view enhancement ${ }^{8,27}$. To capitalize on the rich spatial features of light, frequency upconversion has been utilized for mode-selective detection of spatially orthogonal signals in few-mode waveguides ${ }^{7,28}$, and more recently in nonlinear crystals to selectively convert overlapping Laguerre-Gaussian (LG) and Hermite-Gaussian (HG) modes ${ }^{29,30}$.

In this paper, we further those studies towards field applications and demonstrate mode-selective upconversion and detection of overlapping images mixed with strong turbulence noise. Spatially structured signals, such as those in LG and HG modes, are useful for quantum information processing, remote sensing, and so on ${ }^{12,22,31,32}$. Yet they are quite susceptible to multiscattering and turbulence ${ }^{33-36}$. The ability to distinguish and separately detect or manipulate those disturbed modes is essential to recover the information they carry, as critical for pattern recognition ${ }^{37,38}$, free-space communications ${ }^{7,28,38-41}$, compressive sensing ${ }^{42,43}$, and LiDAR $^{44}$. To this end, a variety of phase compensation methods ${ }^{45,46}$ have been demonstrated using adaptive optics ${ }^{47-50}$, via feedback ${ }^{34}$, feed-forward, or machine learning ${ }^{51,52}$. All of them rely on applying phase or amplitude modulations directly onto the signals, which could induce excessive noise and subject to limited capabilities.

Here, we explore a rather distinct, indirect approach via turbulence-compensating upconversion. Instead of modulating the images ${ }^{34}$, we account for the turbulence using mode-modulated pump to drive the image upconversion, which can select overlapping images despite strong turbulence. This indirect compensation approach avoids any modulation loss or noise added to the signals while allowing more flexible operations.

As a concrete example, here we utilize sum frequency (SF) generation in a lithium niobate crystal, where the signal and pump interact while experiencing strong diffraction ${ }^{27,53,54}$. By preparing the pump in an optimized mode, overlapping signals can be upconverted selectively according to their orthogonal spatial modes. As any turbulence can be described by a unitary linear-optical transformation, distinct modes passing through turbulence will have distorted profiles but will nonetheless remain orthogonal. This allows us to design the pump via adaptive feedback control, to selectively upconvert certain modes over others, even when their intensity profiles are strongly distorted by turbulence to totally unrecognizable. We demonstrate the selective upconversion of one LG modes against another with up to $40 \mathrm{~dB}$ extinction without turbulence, and up to $21 \mathrm{~dB}$ under strong turbulence. Our experimental results match fairly well with numerical simulations without using any fitting parameter.

\section{Theory}

The LG modes can be written in the cylindrical coordinate system as ${ }^{16}$

${ }^{1}$ Department of Physics, Stevens Institute of Technology, Hoboken, NJ, 07030, USA. ${ }^{2}$ Center for Quantum Science and Engineering, Stevens Institute of Technology, Hoboken, NJ, 07030, USA. *email: yuping.huang@stevens.edu 


$$
\begin{aligned}
L G_{l}^{p}(r, \phi, z)= & \frac{C_{l p}}{w(z)}\left(\frac{r \sqrt{2}}{w(z)}\right)^{|l|} \exp \left(\frac{-r^{2}}{w^{2}(z)}\right) \exp (-i k z) L_{p}^{|l|}\left(\frac{2 r^{2}}{w^{2}(z)}\right) \\
& \exp \left(-i k \frac{r^{2}}{2 R(z)}\right) \exp (-i l \phi) \exp (i \zeta(z)),
\end{aligned}
$$

where $r=\sqrt{x^{2}+y^{2}}$ is the radial coordinate, $\phi=\arctan (y / x)$ is the azimuthal coordinate, $C_{l p}=\sqrt{\frac{2 p !}{\pi(p+|l|) !}}$ is a normalization constant, $w(z)=w_{0} \sqrt{1+\left(z / z_{R}\right)^{2}}$ is the beam radius at $z, w_{0}$ is the beam waist, $z_{R}=\pi w_{0}^{2} / \lambda$ is the Rayleigh range, $R(z)=z\left(1+\left(z_{R} / z\right)^{2}\right)$ is the curvature radius of the beam, $\left\{L_{p}|l|\right\}$ are the generalized Laguerre polynomials with the azimuthal mode index $l$ and the radial index $p, k=2 \pi n / \lambda$ is the wave number, and $\zeta(z) \equiv(2 p+|l|+1) \arctan \left(z / z_{R}\right)$ is the Gouy phase at $z$.

The SF is described under the slowly-varying-envelope approximation as:

$$
\begin{aligned}
& 2 i \kappa_{s} \partial_{z} \psi_{s}+\left(\partial_{x}^{2}+\partial_{y}^{2}\right) \psi_{s}=-2 \frac{\omega_{s}^{2}}{c^{2}} \chi^{(2)} \psi_{p}^{*} \psi_{f} e^{i \Delta \kappa z}, \\
& 2 i \kappa_{p} \partial_{z} \psi_{p}+\left(\partial_{x}^{2}+\partial_{y}^{2}\right) \psi_{p}=-2 \frac{\omega_{p}^{2}}{c^{2}} \chi^{(2)} \psi_{s}^{*} \psi_{f} e^{i \Delta \kappa z}, \\
& 2 i \kappa_{f} \partial_{z} \psi_{f}+\left(\partial_{x}^{2}+\partial_{y}^{2}\right) \psi_{f}=-2 \frac{\omega_{f}^{2}}{c^{2}} \chi^{(2)} \psi_{p} \psi_{s} e^{-i \Delta \kappa z},
\end{aligned}
$$

where $\Delta \kappa=\kappa_{s}+\kappa_{p}-\kappa_{f}-2 \pi / \Lambda$ is the momentum mismatching. $\kappa_{s}=\frac{n_{s} \omega_{s}}{c}, \kappa_{p}=\frac{n_{p} \omega_{p}}{c}$ and $\kappa_{f}=\frac{n_{f} \omega_{f}}{c}$ are the wave numbers of signal, pump and SF light, respectively. $\chi^{(2)}$ is the second-order nonlinear susceptibility and $\Lambda$ is the poling period of the nonlinear crystal. $\psi_{s}, \psi_{p}$, and $\psi_{f}$ are the electric fields for the signal, pump, and SF, respectively. The energy in the frequency upconversion process is conserved i.e., $\omega_{p}+\omega_{s}=\omega_{f}$. We use the standard split-step Fourier and adaptive step size methods to numerically solve Eqs. (2)- $(4)^{55}$.

The total SF power is $P_{f}=2 \varepsilon_{0} c n_{f} \int_{0}^{\infty} \int_{0}^{\infty}\left|\psi_{f}(x, y, z)\right|^{2} d x d y$, where $\varepsilon_{0}$ is the permittivity and $\mathrm{c}$ is the speed of light in vacuum. In our experiment, however, the SF light is first coupled into a single mode fiber before detection. This is necessary to achieve both high selectivity and high collection efficiency, as critical for photon-starving and/or cascaded quantum applications ${ }^{44,56}$. The conversion extinction of two modes is then defined as the ratio of their converted SF power coupled into the fiber by the same pump.

To study the turbulence effects, we use the Kolmogorov model to simulate the refractive index variation in the atmospheric turbulence ${ }^{37}$. The power spectral density for the refractive-index fluctuations can be defined as

$$
\Phi_{n}(z, k)=0.0033 c_{n}^{2}(z) k^{-11 / 3},
$$

with $1 / L_{0}<<k<<1 / l_{0}$. Here $k$ is the scalar wave number, $L_{0}$ and $l_{0}$ are the outer and inner scales for the turbulence, respectively and $c_{n}{ }^{2}$ is the structure constant of the atmosphere at the propagation distance $z$. The strength of the turbulence is then approximately defined by Strehl ratio $(S R)$ as

$$
S R=\frac{1}{1+\left(D / r_{0}\right)^{5 / 3}},
$$

where $\mathrm{D}$ is the diameter of the optical beam, $r_{0}$ is the Fried's parameter, with $r_{0}=0.18\left(\lambda^{2} / c_{n}{ }^{2} z\right)^{3 / 534,37}$. SR varies between 0 and 1, with $S R=0$ indicating the maximum turbulence and $S R=1$ no turbulence.

\section{Experimental Set Up}

The experimental setup is shown in Fig. 1. We create the pump and signal pulses by electro-optically modulating the outputs of continuous lasers, each at $1544.9 \mathrm{~nm}$ and $1558.5 \mathrm{~nm}$. Those pulses are synchronized to a common reference radio-frequency source. Each pulse has a 200-ps full width at half maximum (FWHM) and $10-\mathrm{MHz}$ repetition rate. The pulses are amplified using two separate Erbium-Doped Fiber Amplifiers (EDFAs). The pump's average power is $40 \mathrm{~mW}$ and peak power is $20 \mathrm{~W}$. The signal's average power is $35 \mathrm{~mW}$ and peak power is $17.5 \mathrm{~W}$. We use a half waveplate (HWP) and a quarter waveplate (QWP) with polarizing beam splitters (PBS's) on both arms to select the horizontal polarization for the pump and signal beams. The beams are then magnified by telescopes to $2.6 \mathrm{~mm}$ FWHM for the signal and $2.8 \mathrm{~mm}$ for the pump. Afterwards, they are incident on SLMs (Santec SLM-100) with the angle of incidences of $50^{\circ}$ and $55^{\circ}$, respectively ${ }^{29}$. The total phase pattern for converting the input Gaussian beam into the $L G_{l} p$ beam is given as $\Theta(r, \phi)=-l \phi+\pi \theta\left(-L_{p}^{|l|}\left(\frac{2 r^{2}}{\omega_{0}^{2}}\right)\right)$, with $\theta$ as a unit step function. The phase value is wrapped in the interval between 0 and $2 \pi$ to express on the SLMs.

The signal and pump beams are then combined at a beam splitter (BS) and focused $(f=200 \mathrm{~mm})$ inside a temperature-stabilized PPLN crystal with a poling period of $19.36 \mu \mathrm{m}$ (5 mol.\% MgO doped PPLN, $10 \mathrm{~mm}$ length, $3 \mathrm{~mm}$ width, and $1 \mathrm{~mm}$ height from HC Photonics) for frequency conversion process. HWP is used to ensure the vertically polarized light parallel to the crystal's optical axis. The beam waist of the signal and pump, both in Gaussian spatial modes, inside the crystal are $45 \mu \mathrm{m}$ and $41 \mu \mathrm{m}$, respectively. We also fine tune the time 


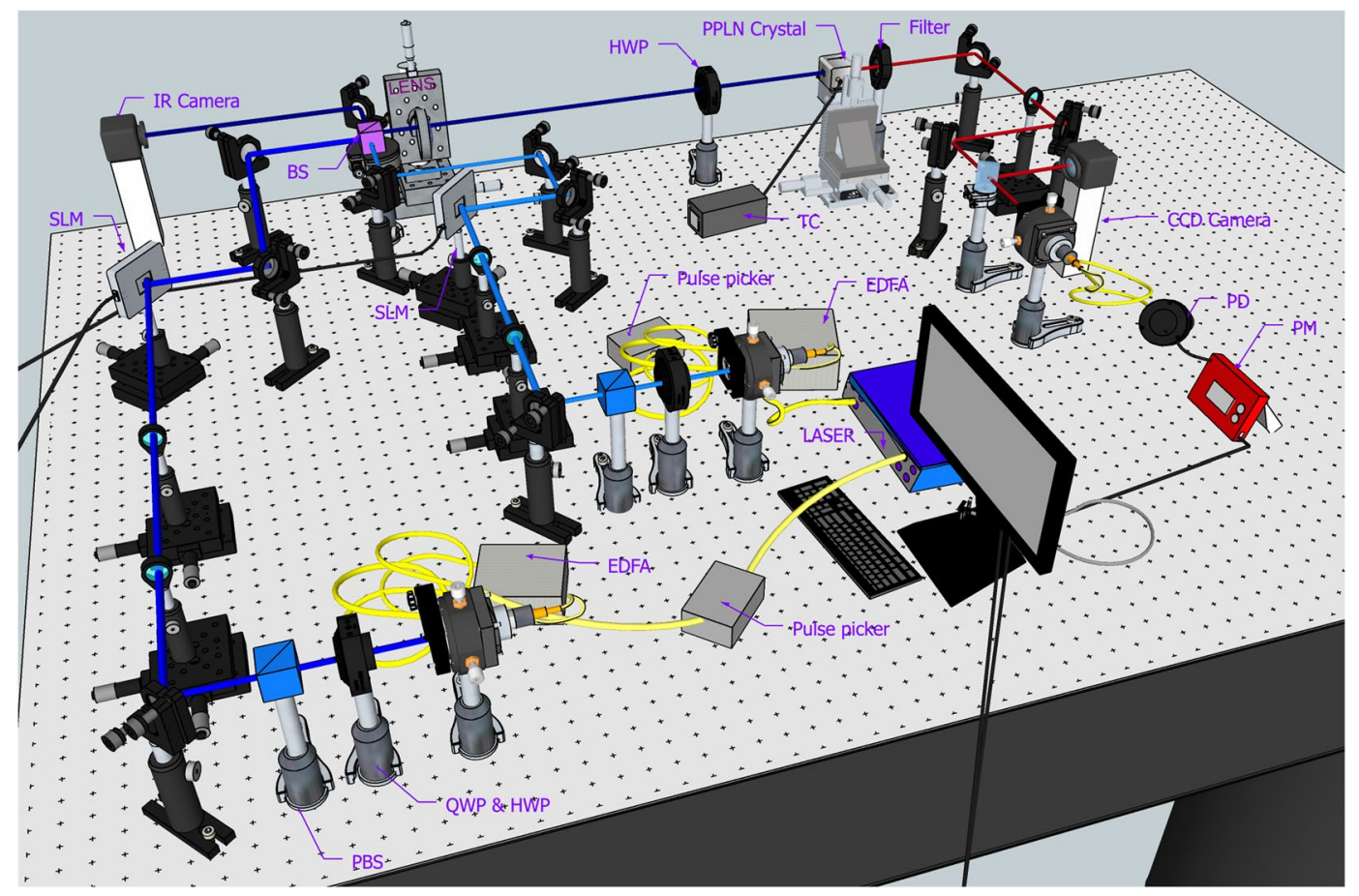

Figure 1. Experimental setup. Two synchronized light pulse trains, each at 1544.9 and $1558.5 \mathrm{~nm}$, are created through commonly referenced pulse pickers. The SLMs are used to create the desired spatial modes. The pulse trains are combined using a BS and passed through a temperature stabilized PPLN crystal. The generated SF light at $775.85 \mathrm{~nm}$ is filtered and coupled into a single mode fiber for detection using a power meter. Its result is fed via MATLAB to adaptively optimize the phase mask on pump SLM. EDFA: Erbium-doped fiber amplifier, QWP: Quarter waveplate, HWP: Half waveplate, BS: Beamsplitter, SLM: Spatial Light Modulator, PPLN crystal: Magnesium-doped periodic poled lithium niobate crystal, PD: Photodiode, PM: Powermeter, TC: Temperature Controller.

delay between the signal and pump arm to obtain good temporal overlap of their pulses. The output is then passed through two short-pass filters to select the SF light and remove any residual light ${ }^{24}$. The central wavelength of the SF light is $775.85 \mathrm{~nm}$ with FWHM of $0.05 \mathrm{~nm}$. The conversion efficiency of SF generation process for Gaussian signal and Gaussian pump is $2.2 \%$. As the order increases, the power density of LG mode decreases, which gives rise to the lower conversion efficiency ${ }^{57}$. This makes it more difficult to selectively up-convert higher-order LG modes. The other arm of the BS is used to monitor the spatial mode of the pump and signal beams using a near-infrared FIND-R-SCOPE camera. The same arm can also be used to monitor the intensity fluctuation on an high speed oscilloscope (not shown in the figure). The SF light is split in two parts using a flip-able BS. A lens placed at one arm of the flip-able BS is used to image the collimated SF light on a CCD camera with a sensor size of $22.3 \mathrm{~mm} \times 14.9 \mathrm{~mm}$ and a pixel pitch of $4.3 \mu \mathrm{m}$. The other arm of the SF light is coupled into a single mode fiber (with coupling efficiency $\sim 60 \%$ ) and detected by the power meter sensor (Thorlabs PM-100D with sensor S130C). This measurement is sent to the computer via a MATLAB interface for the adaptive feedback control process. It updates the phase mask on pump SLM to optimize the selectivity among the signal modes. The effect of the turbulence on the signal is simulated by adding turbulence phase noises to the original phase mask for the LG modes.

\section{Results and Discussions}

To illustrate the turbulence effects, in Fig. 2 we plot the resulting LG modes at $1558.5 \mathrm{~nm}$ under turbulence of different strengths. From Eq. (6), as $S R$ decreases, the strength of the turbulence increases, and the spatial coherence degrades to give distorted intensity distribution. At $S R=0.3$, all modes become illegible. In the figure, the appearance of narrow outer rings is a consequence of the phase only modulation ${ }^{58}$.

In Fig. 3 we show the spatial profiles of the upconverted SF lights for different combinations of signal and pump $L G_{l}^{p}$ modes without turbulence $(S R=1)$. The numerical results in Fig. 3(a) are evaluated by solving Eqs. $(2-4)$ for $\left|\psi_{f}(x, y)\right|^{2}$. In a previous work, we have studied the selective upconversion of overlapping spatial modes in the non-diffraction regime with the same helicity for the signal and pump beams ${ }^{29}$. Here, we consider azimuthal indices from $+l$ to $-l$ for both signal and pump beams. When $l_{p}=-l_{s}$, the SF mode has a central Gaussian bright spot and thick outer rings, as expected. The Insets of Fig. 3 show the SF modes for a Gaussian pump with turbulent signal modes. As seen, turbulence significantly reduce the total power and distort the intensity profiles of the generated SF modes. Our simulated SF modes exhibit similar features with the measured results.

Unlike previous studies where the SF power were directly measured at an image plane ${ }^{7,29,30}$, here we couple the SF lights into a single mode fiber. The fiber acts as a spatial mode filter on the SF light to improve to detection extinction ${ }^{59-61}$, while also providing convenience for subsequent processing of the SF light. For instance, by 

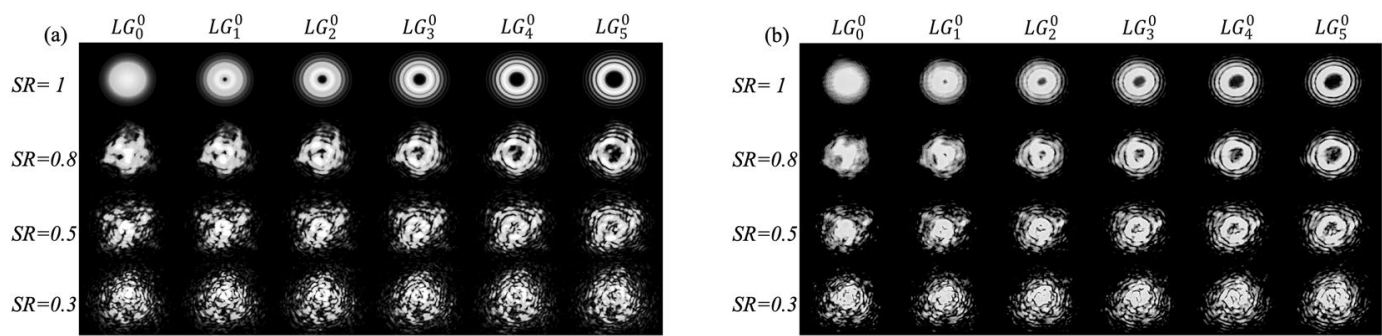

Figure 2. (a) Simulated and (b) measured signal modes without and with varied turbulence.

(a)

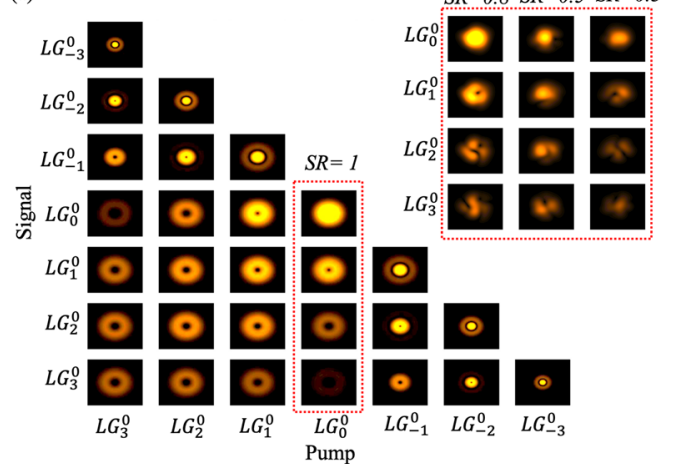

(b)

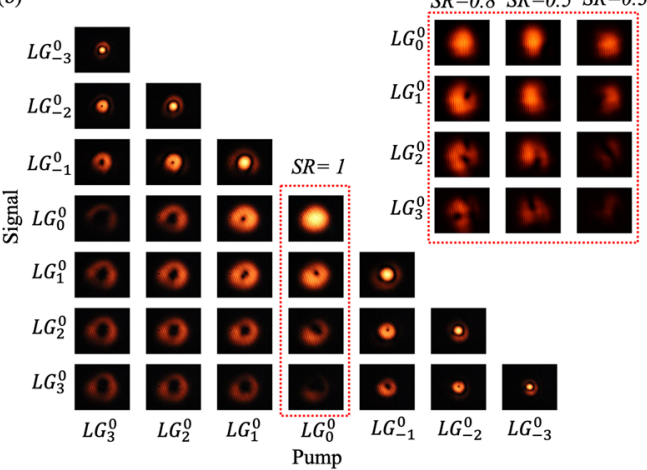

Figure 3. (a) Numerical and (b) experimental results of the generated SF modes with no turbulence on both signal and pump. Insets of (a) and (b) show the SF modes generated by Gaussian pumps but turbulencedistorted signals.

measuring the total SF power, our previous experiment achieved $\sim 5.6 \mathrm{~dB}$ selectivity between two signal modes $L G_{0}{ }^{0}$ and $L G_{1}{ }^{0}$ with an un-optimized pump in mode $L G_{0}{ }^{029}$. With the same pump, the extinction is increased to $15.9 \mathrm{~dB}$ by measuring the fiber-coupled SF power. In the current setup, the fiber coupling efficiency is $\sim 60 \%$ for the SF light created by a Gaussian signal and a Gaussian pump. In comparison, in a recent study ${ }^{30}$, the extinction was derived by selecting the only on-axis SF intensity of the central pixel on a CCD camera. Thus the effective detection efficiency is rather low. In contrast, the current setup can achieve both high extinction and high detection efficiency at the same time, which is critical for classical and quantum communications in practical settings.

To further improve the selectivity, the particle swarm optimization algorithm is used to numerically optimize the pump spatial profiles for the target signal modes ${ }^{29}$. In this method, the optimum pump shape is produced as a superposition of LG mode masks. We then apply these numerically optimized phase masks on SLM to create the pump. However, due to the inevitable phase errors in the SLM and imperfect alignment of the optical beam, the measured selectivity is significantly lower than achievable in simulation. To mitigate the errors and imperfections, we apply an adaptive feedback loop using a random-walk optimization algorithm in our experiment to fine tune the pump's phase masks. For more information, please refer to our previous work where the same feedback method was carried out for mode selective conversion without turbulence ${ }^{29}$.

Table 1 shows the selectivity performance for the target spatial mode $L G_{0}{ }^{0}$ over other orthogonal modes, where $\xi_{i}$ the $\mathrm{dB}$ ratio of fiber-coupled SF power of the $L G_{0}{ }^{0}$ mode to $L G_{i}{ }^{0}$ mode with the same pump. Here, the pump $S\left[L G_{0}{ }^{0}\right]^{S R}$ is optimized to selectively upconvert signal mode $L G_{0}{ }^{0}$ while simultaneously suppressing $L G_{1}{ }^{0}, L G_{2}{ }^{0}, L G_{3}{ }^{0}, L G_{4}{ }^{0}$, and $L G_{5}{ }^{0}$ without turbulence $(S R=1)$, and with the same turbulence noise $(S R=0.5)$. In numerical simulations, without turbulence the extinction reaches $44.4 \mathrm{~dB}$, while the best experimental result gives $39.1 \mathrm{~dB}$. As we add the turbulence, the selectivity obtained using the same pump $S\left[L G_{0}{ }^{0}\right]^{S R=1}$ drops significantly, with the extinction reduced by about $23 \mathrm{~dB}$ on average in simulation, and up to $18 \mathrm{~dB}$ in experiment. This signifies the strong distortion of the signal modes by the turbulence. To overcome it, we then create re-optimized pump mode $S\left[L G_{0}{ }^{0}\right]^{S R=0.5}$ using the same feedback method to recover the high extinctions. As a result, the extinctions increase significantly by as high as $24 \mathrm{~dB}$ in simulation and $15 \mathrm{~dB}$ in experiment. This improvement is on par with that of a linear-optics approach where adaptive phase compensation is directly applied to the signal with a SLM ${ }^{34}$. Without turbulence the minimum extinction ratio for high-order orbital angular momentum, in their work, is around $15.28 \mathrm{~dB}$. With similar turbulence strength, the minimum extinction ratios before and after compensation are -1.2 and $13.82 \mathrm{~dB}$, respectively. Thus the adaptive linear-optics methods and our indirect nonlinear method have comparable extinctions. However, the indirect modulation method has advantages that we can avoid any modulation loss or noise added to the signals. It is capable of compensating for both phase and amplitude distortions at the same time.

Note that in experiment, while the re-optimization significantly improves the measured extinction for the first LG modes, it gives less improvement on the higher-order LG modes. This is because our current optimization 


\begin{tabular}{|l|l|l|l|l|l|l|}
\hline & $S R$ & $\begin{array}{l}\xi_{1} \\
(\mathbf{d B})\end{array}$ & $\begin{array}{l}\xi_{2} \\
(\mathbf{d B})\end{array}$ & $\begin{array}{l}\xi_{3} \\
(\mathbf{d B})\end{array}$ & $\begin{array}{l}\xi_{4} \\
(\mathbf{d B})\end{array}$ & $\begin{array}{l}\xi_{5} \\
(\mathbf{d B})\end{array}$ \\
\hline$(\mathbf{a})$ & 1 & 27.3 & 25.3 & 32.7 & 35.7 & 44.4 \\
\hline \multirow{2}{*}{$S\left[L G_{0}{ }^{0}\right]^{S R=1}$} & 0.5 & 2.1 & 7.0 & 10.2 & 11.8 & 18.6 \\
\hline$S\left[L G_{0}{ }^{0}\right]^{S R=0.5}$ & 0.5 & 26.1 & 21.8 & 17.8 & 23.0 & 31.8 \\
\hline$(\mathbf{b})$ & 1 & 27.2 & 27.6 & 29.6 & 30.5 & 39.1 \\
\hline$S\left[L G_{0}{ }^{0}\right]^{S R=1}$ & 0.5 & 4.7 & 9.9 & 14.3 & 15.5 & 19.2 \\
\hline$S\left[L G_{0}{ }^{0}\right]^{S R=0.5}$ & 0.5 & 20.1 & 19.2 & 18.8 & 20.7 & 19.3 \\
\hline
\end{tabular}

Table 1. (a) Simulated and (b) experimental results for the selective upconversion of the signal modes using optimized pump with $(S R=0.5)$ and without turbulence $(S R=1) . \xi_{i}$ is the extinction of selecting the $L G_{0}{ }^{0}$ over $L G_{i}^{0}$ mode.
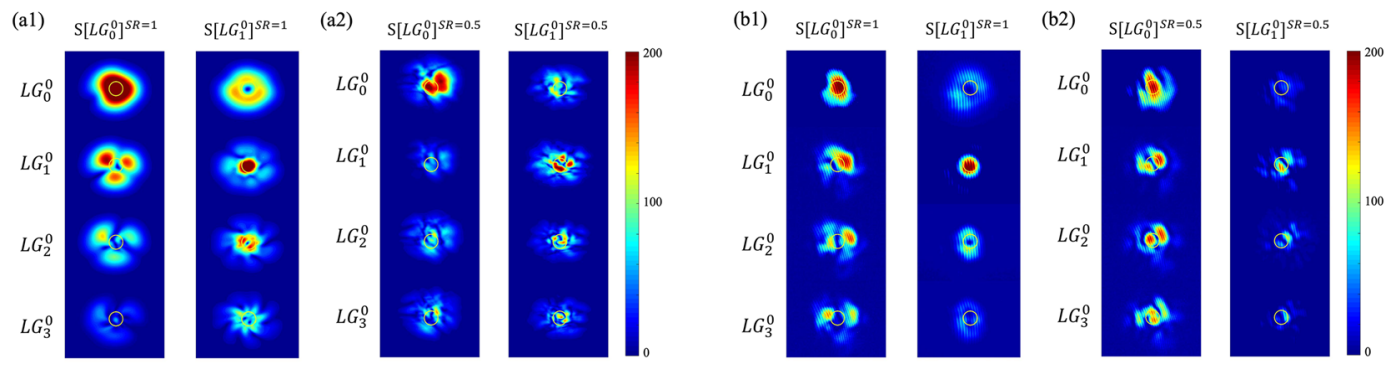

Figure 4. (a1,a2) Numerical and (b1,b2) experimental results for SF modes created by optimized pumps. In (a1) and (b1), the pump $S\left[L G_{0}{ }^{0}\right]^{S R=1}$ and $S\left[L G_{1}{ }^{0}\right]^{S R=1}$ are optimized to selectively upconvert the signal modes $L G_{0}{ }^{0}$ and $L G_{1}{ }^{0}$, respectively, with no turbulence $(S R=1)$. In (a2) and (b2), the pump $S\left[L G_{0}^{0}\right]^{S R=0.5}$ and $S\left[L G_{1}{ }^{0}\right]^{S R=0.5}$ are optimized to selectively upconvert signal modes $L G_{0}{ }^{0}$ and $L G_{1}{ }^{0}$, respectively, with strong turbulence $S R=0.5$. The yellow circle indicated the coupling region into a single mode fiber.

method emphasizes on increasing the lowest extinction to achieve high extinction of the $L G_{0}{ }^{0}$ mode over all of the other LG modes. As the extinction against those higher-order modes remain high despite turbulence, the re-optimization improves them only marginally. In practice, higher performance can be achieved by tailoring the optimization to take advantage of any prior information of the modes to be detected.

To understand those re-optimization results, in Fig. 4 we plot the resulting spatial profile of the SF modes with the optimized pump. Here, the optimized pumps $S\left[L G_{i}{ }^{0}\right]^{S R}$ is designed to selectively upconvert $L G_{i}{ }^{0}$ while simultaneously suppressing all others three modes, for the cases of with turbulence $(S R=0.5)$ and without turbulence $(S R=1)$. The yellow circle highlights the effective region of the SF light coupled into the single mode fiber. As seen, the (re-)optimization significantly increases the optical power inside the fiber-coupled mode area for the target mode, but not the others. Our experimental results for the optimized pump qualitatively agree with the simulations.

Figure 5 presents a series of examples on the selective upconversion of spatial modes with re-optimized pump for various turbulence. Figure 5(a1-a4) display the phase masks added to the phase pattern of the LG modes to simulate the turbulence effects. Figure 5(b,c) compare the numerical and experimental extinctions for selectively upconverting $L G_{0}{ }^{0}$ and $L G_{1}{ }^{0}$ modes, respectively, under various turbulence. As shown in Fig. 5(b1-b4), when the turbulence increases, the extinction drops. However, even for quite strong turbulence at $\mathrm{SR}=0.3$, the average extinction still reaches an average of $16 \mathrm{~dB}$ and $13 \mathrm{~dB}$ for numeric and experimental results, respectively. For the $L G_{1}{ }^{0}$ mode, we obtain similar results, as shown in Fig. 5(c1-c4), with the average extinction drops to $12 \mathrm{~dB}$ and $9 \mathrm{~dB}$ in simulation and experiment.

The above shows the results to selectively upconvert a certain mode over others. With straightforward modification of the convergence condition in the optimization algorithm, the same technique can be employed for other operations, including mode deselection, where a certain mode is dropped, rather than picked, from other overlapping modes.

Figure 6 shows some de-selection results in the presence of different turbulent strengths. Opposite to $S\left[L G_{0}{ }^{0}\right]^{S R}$ for selection, now the optimized pump $D\left[L G_{0}{ }^{0}\right]^{S R}$ is designed to avoid converting one undesired mode while increasing converting other overlapping modes with high efficiency. Figure 6(b) shows the simulated and measured extinctions for the signal modes by using individually optimized pump modes. Here, a large negative extinction means a much lower conversion efficiency of the target mode versus others, which is desirable. In Fig. 6, the optimized pump $D\left[L G_{1}{ }^{0}\right]^{S R=1}$ gives the numerically evaluated extinctions $-33.6 \mathrm{~dB},-31.2 \mathrm{~dB}$, $-24.8 \mathrm{~dB},-29.0 \mathrm{~dB}$, and $-24.2 \mathrm{~dB}$ and experimentally observed extinctions $-26.3 \mathrm{~dB},-24.4 \mathrm{~dB},-20.2 \mathrm{~dB}$, $-17.7 \mathrm{~dB}$, and $-11.9 \mathrm{~dB}$ for the signal modes $L G_{1}{ }^{0}, L G_{2}{ }^{0}, L G_{3}{ }^{0}, L G_{4}{ }^{0}$, and $L G_{5}{ }^{0}$, respectively. In experiment, the use of re-optimized pump modes for turbulence give extinctions of (b2) $-23.7 \mathrm{~dB},-21.6 \mathrm{~dB},-18.4 \mathrm{~dB},-19.0 \mathrm{~dB}$, 

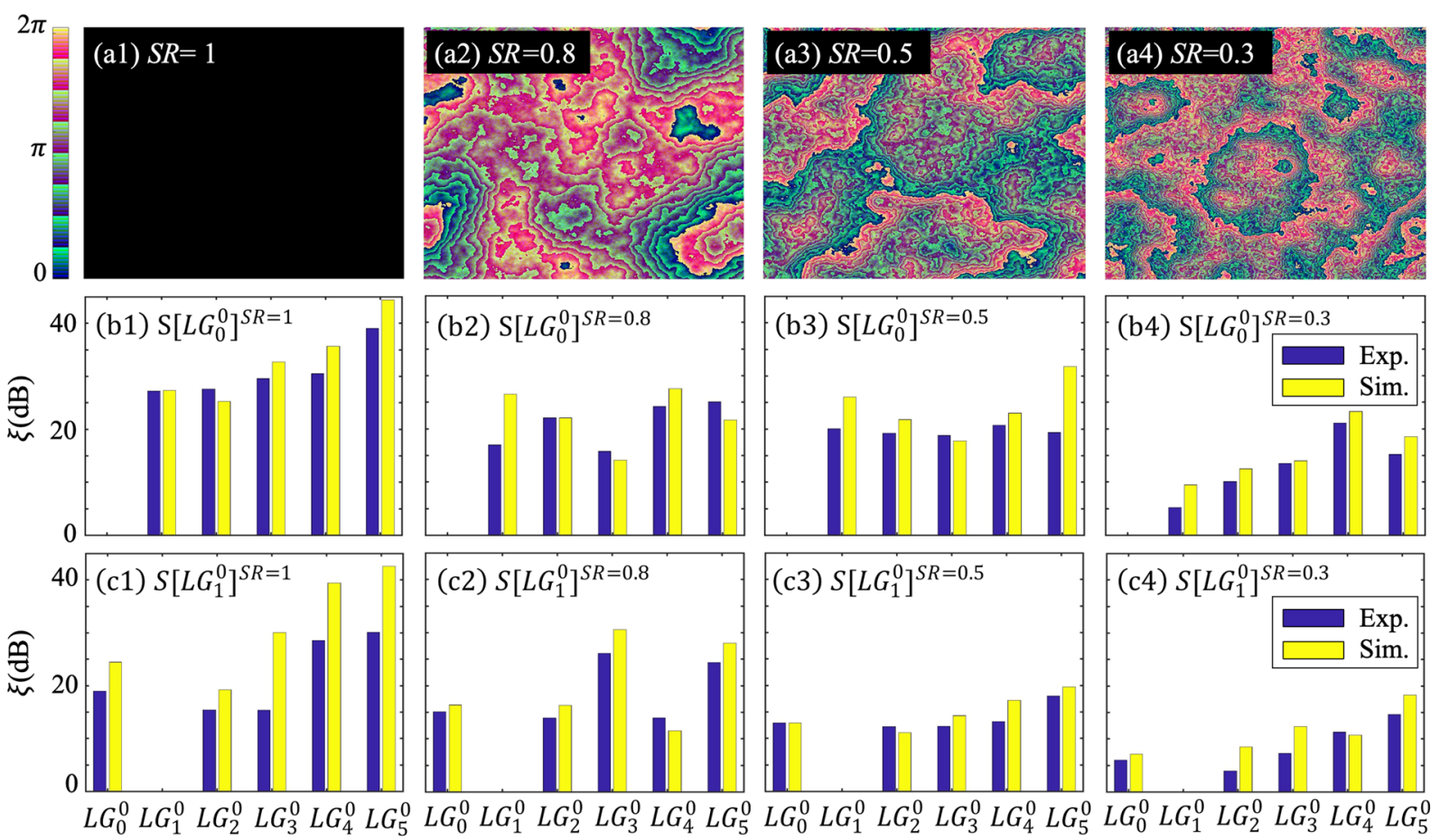

Figure 5. (a1-a4) Phase masks to simulate various level of turbulence. (b1-b4) Experimental (blue bars) and simulated (yellow bars) results with the (re)-optimized pump $S\left[L G_{0}{ }^{0}\right]^{S R}$ to select $L G_{0}{ }^{0}$ signal mode while suppressing other modes under those turbulence. (c1-c4) are similar results but to select $L G_{1}{ }^{0}$.
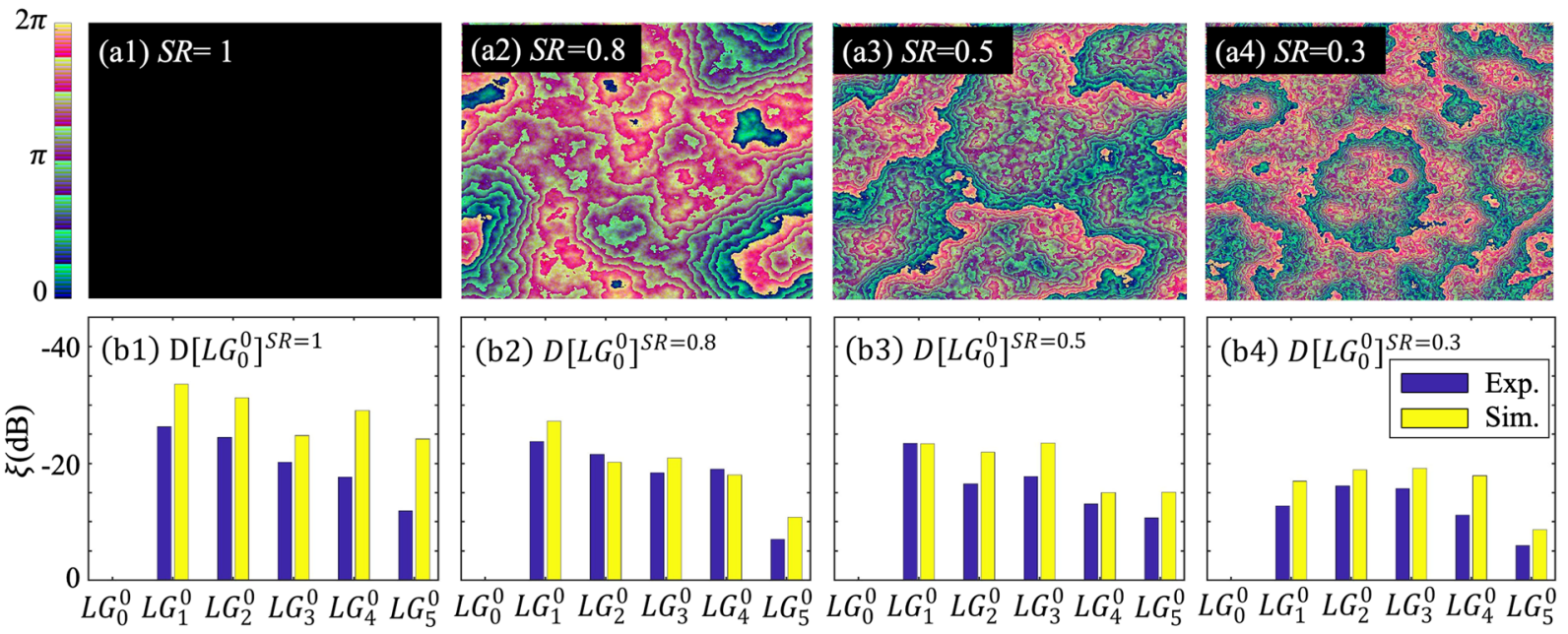

Figure 6. Similar to Fig. 5 but with the pump optimized to de-select the $L G_{0}{ }^{0}$ signal mode.

and $-7.0 \mathrm{~dB}$ for $S R=0.8$; (b3) $-23.5 \mathrm{~dB},-16.5 \mathrm{~dB},-17.7 \mathrm{~dB},-13.0 \mathrm{~dB}$, and $-10.7 \mathrm{~dB}$ for $S R=0.5$; and (b4) $-12.7 \mathrm{~dB},-16.2 \mathrm{~dB},-15.7 \mathrm{~dB},-11.1 \mathrm{~dB}$, and $-3.9 \mathrm{~dB} S R=0.3$, respectively. In comparison, simulated results are (b2) $-26.5 \mathrm{~dB},-22.1 \mathrm{~dB},-14.1 \mathrm{~dB},-27.5 \mathrm{~dB}$, and $-21.7 \mathrm{~dB}$; (b3) $-26.1 \mathrm{~dB},-21.8 \mathrm{~dB},-17.8 \mathrm{~dB},-23.0 \mathrm{~dB}$, and $-31.8 \mathrm{~dB}$; (b4) $-9.5 \mathrm{~dB},-12.5 \mathrm{~dB},-14.0 \mathrm{~dB},-23.3 \mathrm{~dB}$, and $-18.5 \mathrm{~dB}$ for $S R=0.8,0.5$ and 0.3 , respectively. Those results highlight good deselection performance.

All of our experimental results are in good agreement with the numerical simulations. In the case of strong turbulence, the extinctions are less recovered by pump re-optimization. This is because the initial fiber-coupled SF power is significantly less, so that the feedback control algorithm works less well. This can be improved in the future with more robust algorithms, or by pushing up the conversion efficiency similarly to our previous work in the time-frequency domain ${ }^{24}$. For practical applications under fast changing turbulence, the current SLM needs to be replaced by a high-speed phase mask controller, such as a FPGA-controlled MEMS-SLM ${ }^{62}$ or digital micromirror device (DMD). These will be subjects of our future studies. 


\section{Conclusion}

In conclusion, we have numerically and experimentally demonstrated mode selective up-conversion of structured spatial modes mixed with turbulent noise. Unlike any existing adaptive optics method that applies compensating modulation directly to the signal, here the turbulence is accounted for indirectly by modultating the pump modes that drives the upconverison, but not the signal. Hence, it fundamentally eliminates the signal modulation noise or loss, while also allowing exceptional flexibility and capabilities in manipulating and detecting overlapping signals, including quantum light at a single photon level. Our experimental results have achieved $\sim 40 \mathrm{~dB}$ extinction under no turbulence, and $\sim 20 \mathrm{~dB}$ even in the presence of strong turbulence. The latter is expected to be significantly increased by using better optimization algorithm and higher pump power to enhance the conversion, which will be a subject of our future studies.

We have also demonstrated the selection and de-selection of a particular mode amongst overlapping modes. The same technique applies to superposition modes, as they correspond a new set of modes in different spatial profiles. Favorably, any signal mode that are not converted will remain in its original wavelength and quantum state. They can thus be recycled for cascaded operations to realize complex functionalities. For example, by using a serial or optical-loop setup similar to that $\mathrm{in}^{21}$, efficient quantum state tomography can be implemented on signals in high-order optical angular momentum states ${ }^{63}$. All of those unique capabilities may prove useful for remote sensing ${ }^{64}$, phase encryption and decryption ${ }^{65}$, compressive imaging ${ }^{42,43}$, quantum free-space optical communication ${ }^{66}$, and so on.

Received: 2 September 2019; Accepted: 28 October 2019;

Published online: 25 November 2019

\section{References}

1. Giordmaine, J. \& Miller, R. Tunable coherent parametric oscillation in linbo 3 at optical frequencies. Phys. Rev. Lett. 14, 973 (1965).

2. Rodrigues, R. B. et al. Orbital angular momentum symmetry in a driven optical parametric oscillator. Opt. Lett. 43, 2486-2489 (2018).

3. Zhou, Z. et al. Orbital angular momentum photonic quantum interface. Light Sci. Appl. 5, e16019 (2016).

4. Sua, Y. M., Fan, H., Shahverdi, A., Chen, J. \& Huang, Y.-P. Direct generation and detection of quantum correlated photons with 3.2 um wavelength spacing. Sci. Rep. 7, 17494 (2017).

5. Delaubert, V., Lassen, M., Pulford, D. R. N., A. Bachor, H. \& Harb, C. C. Spatial mode discrimination using second harmonic generation. Opt. Express 15, 5815-5826 (2007).

6. Pereira, L. J., Buono, W. T., Tasca, D. S., Dechoum, K. \& Khoury, A. Z. Orbital-angular-momentum mixing in type-ii secondharmonic generation. Phys. Rev. A 96, 053856 (2017).

7. Vasilyev, M., Kwon, Y. B. \& Huang, Y.-P. Spatial-mode-selective quantum frequency conversion in a $\chi^{(2)}$ slab waveguide. OSA Technical Digest (online), paper JWA.52 2 (2014).

8. Maestre, H., Torregrosa, A. J., Fernández-Pousa, C. R. \& Capmany, J. Ir-to-visible image upconverter under nonlinear crystal thermal gradient operation. Opt. Express 26, 1133-1144 (2018).

9. Boyer, V., Marino, A. M., Pooser, R. C. \& Lett, P. D. Entangled Images from Four-Wave Mixing. Science 321, 544, https://doi. org/10.1126/science.1158275 (2008).

10. Ding, D. et al. Experimental up-conversion of images. Phys. Rev. A 86, 033803 (2012).

11. Boyd, R. Nonlinear Optics. (Academic Press, USA, 2003).

12. Lassen, M. et al. Tools for multimode quantum information: Modulation, detection, and spatial quantum correlations. Phys. Rev. Lett. 98, 083602 (2007).

13. Yue, S., Slipchenko, M. N. \& X. Cheng, J. Multimodal nonlinear optical microscopy. Laser Photon Rev. 5, 4 (2011).

14. Glasser, R. T. et al. Entanglement-seeded, dual, optical parametric amplification: Applications to quantum imaging and metrology. Phys. Rev. A 78, 012339, https://doi.org/10.1103/PhysRevA.78.012339 (2008).

15. Hallett, D. et al. Electrical control of nonlinear quantum optics in a nano-photonic waveguide. Optica 5, 644-650, https://doi. org/10.1364/OPTICA.5.000644 (2018).

16. Lanning, R. N. et al. Gaussian-beam-propagation theory for nonlinear optics involving an analytical treatment of orbital-angularmomentum transfer. Phys. Rev. A 96, 013830 (2017).

17. Demur, R. et al. Near-infrared to visible upconversion imaging using a broadband pump laser. Opt. Express 26, 13252-13263 (2018).

18. Benjamin, B., Eckstein, A., Christ, A., Suche, H. \& Silberhorn, C. From quantum pulse gate to quantum pulse shaper-engineered frequency conversion in nonlinear optical waveguides. New Jour. of Phys. 13, 65029 (2011).

19. Eckstein, A., Brecht, B. \& Silberhorn, C. Quantum pulse gate based on spectrally engineered sum frequency generation. Opt. Express 19, 13770-13778 (2011).

20. Reddy, D. V., Raymer, M. G., McKinstrie, C. J., Mejling, L. \& Rottwitt, K. Temporal mode selectivity by frequency conversion in second-order nonlinear optical waveguides. Opt. Express 21, 13840-13863 (2013).

21. Huang, Y.-P. \& Kumar, P. Mode-resolved photon counting via cascaded quantum frequency conversion. Opt. Lett. 38, 468-470 (2013).

22. Brecht, B., Reddy, D., Silberhorn, C. \& Raymer, M. Photon temporal modes: A complete framework for quantum information science. Phys. Rev. X 5, 041017 (2015).

23. Allgaier, M. et al. Highly efficient frequency conversion with bandwidth compression of quantum light. Nat. Commun. 8, 14288 (2017).

24. Shahverdi, A., Sua, Y. M., Tumeh, L. \& Huang, Y.-P. Quantum parametric mode sorting: Beating the time-frequency filtering. Sci. Rep. 7, 6495 (2017).

25. Köprülü, K. G., Huang, Y.-P., Barbosa, G. A. \& Kumar, P. Lossless single-photon shaping via heralding. Opt. Lett. 36, 1674-1676, https://doi.org/10.1364/OL.36.001674 (2011).

26. Qiu, X., Li, F., Zhang, W., Zhu, Z. \& Chen, L. Spiral phase contrast imaging in nonlinear optics: seeing phase objects using invisible illumination. Optica 5, 208-212 (2018).

27. Liu, S.-K. et al. Up-Conversion Imaging Processing With Field-of-View and Edge Enhancement. Phys. Rev. Appl. 11, 044013, https:// doi.org/10.1103/PhysRevApplied.11.044013 (2019).

28. Kwon, Y. B., Giribabu, M., Langrock, C., Fejer, M. M. \& Vasilyev, M. Single-photon-level spatial-mode-selective frequency upconversion in a multimode $\chi^{(2)}$ waveguide. OSA Tech. Dig. (online), paper FFE. 22 (2017).

29. Kumar, S., Zhang, H., Maruca, S. \& Huang, Y.-P. Mode-selective image upconversion. Opt. Lett. 44, 98-101 (2019).

30. Sephton, B. et al. Spatial mode detection by frequency upconversion. Opt. Lett. 44, 586-589, https://doi.org/10.1364/OL.44.000586 (2019). 
31. Pang, K. et al. Demonstration of a $10 \mathrm{Mbit} / \mathrm{s}$ quantum communication link by encoding data on two laguerre-gaussian modes with different radial indices. Opt. Lett. 43, 5639-5642, https://doi.org/10.1364/OL.43.005639 (2018).

32. Fontaine, N. K. et al. Laguerre-Gaussian mode sorter. Nat. Commun. 10, 1865, https://doi.org/10.1038/s41467-019-09840-4 (2019).

33. Malik, M. et al. Influence of atmospheric turbulence on optical communications using orbital angular momentum for encoding. Opt. Express 20, 13195-13200, https://doi.org/10.1364/OE.20.013195 (2012).

34. Li, S., Chen, S., Gao, C., Willner, A. E. \& Wang, J. Atmospheric turbulence compensation in orbital angular momentum communications: Advances and perspectives. Opt. Commun. 408, 68-81, https://doi.org/10.1016/j.optcom.2017.09.034 (2018).

35. Zhou, M., Zhou, Y., Wu, G. \& Cai, Y. Reducing the cross-talk among different orbital angular momentum modes in turbulent atmosphere by using a focusing mirror. Opt. Express 27, 10280-10287, https://doi.org/10.1364/OE.27.010280 (2019).

36. Rubinsztein-Dunlop, H. et al. Roadmap on structured light. Journal of Optics 19, 013001, https://doi.org/10.1088/20408978/19/1/013001 (2017)

37. Kolmogorov, A. N., Levin, V., Hunt, J. C. R., Phillips, O. M. \& Williams, D. The local structure of turbulence in incompressible viscous fluid for very large reynolds numbers. Proc. Royal Soc. London. Ser. A: Math. Phys. Sci. 434, 9-13, https://doi.org/10.1098/ rspa.1991.0075 (1991).

38. Ren, Y. et al. Adaptive-optics-based simultaneous pre- and post-turbulence compensation of multiple orbital-angular-momentum beams in a bidirectional free-space optical link. Optica 1, 376-382, https://doi.org/10.1364/OPTICA.1.000376 (2014).

39. Sit, A. et al. High-dimensional intracity quantum cryptography with structured photons. Optica 4, 1006-1010, https://doi. org/10.1364/OPTICA.4.001006 (2017).

40. Bozinovic, N. et al. Terabit-scale orbital angular momentum mode division multiplexing in fibers. Science 340, 1545-1548 (2013).

41. Huang, H. et al. Mode division multiplexing using an orbital angular momentum mode sorter and mimo-dsp over a graded-index few-mode optical fibre. Sci. Rep. 5, 14931 (2015).

42. Howland, G. A. \& Howell, J. C. Efficient high-dimensional entanglement imaging with a compressive-sensing double-pixel camera. Phys. Rev. X 3, 011013, https://doi.org/10.1103/PhysRevX.3.011013 (2013).

43. Cai, X., Hu, B., Sun, T., Kelly, K. F. \& Baldelli, S. Sum frequency generation-compressive sensing microscope. The J. Chem. Phys. 135, 194202, https://doi.org/10.1063/1.3660202 (2011).

44. Shahverdi, A., Sua, Y. M., Dickson, I., Garikapati, M. \& Huang, Y.-P. Mode selective up-conversion detection for lidar applications. Opt. Express 26, 15914-15923 (2018).

45. Qassim, H. et al. Limitations to the determination of a Laguerre-Gauss spectrum via projective, phase-flattening measurement. J. Opt. Soc.Am. B 31, A20, https://doi.org/10.1364/JOSAB.31.000A20 (2014).

46. Mair, A., Vaziri, A., Weihs, G. \& Zeilinger, A. Entanglement of the orbital angular momentum states of photons. Nature 412, 313-316, https://doi.org/10.1038/35085529 (2001).

47. Krenn, M. et al. Communication with spatially modulated light through turbulent air across Vienna. New J. Phys. 16, 113028, https:// doi.org/10.1088/1367-2630/16/11/113028 (2014).

48. Rodenburg, B. et al. Simulating thick atmospheric turbulence in the lab with application to orbital angular momentum communication. New J. Phys. 16, 033020, https://doi.org/10.1088/1367-2630/16/3/033020 (2014).

49. Gemayel, P., Colicchio, B., Dieterlen, A. \& Ambs, P. Cross-talk compensation of a spatial light modulator for iterative phase retrieval applications. Appl. Opt. 55, 802-810, https://doi.org/10.1364/AO.55.000802 (2016).

50. Li, S. \& Wang, J. Adaptive free-space optical communications through turbulence using self-healing Bessel beams. Sci. Rep. 7, 43233, https://doi.org/10.1038/srep43233 (2017)

51. Lohani, S. \& Glasser, R. T. Turbulence correction with artificial neural networks. Opt. Lett. 43, 2611-2614, https://doi.org/10.1364/ OL.43.002611 (2018).

52. Liu, J. et al. Deep learning based atmospheric turbulence compensation for orbital angular momentum beam distortion and communication. Opt. Express 27, 16671-16688, https://doi.org/10.1364/OE.27.016671 (2019).

53. Li, Y., Zhou, Z.-y, Ding, D.-s \& Shi, B.-s Sum frequency generation with two orbital angular momentum carrying laser beams. J. Opt. Soc. Am. B 32, 407-411 (2015).

54. Li, Y., Zhou, Z.-Y., Ding, D.-S. \& Shi, B.-S. Dynamic mode evolution and phase transition of twisted light in nonlinear process. J. Mod. Opt. 63, 2271-2278, https://doi.org/10.1080/09500340.2016.1197333 (2016).

55. Agrawal, G. Nonlinear Fiber Optics (Fifth Edition) (Academic Press, Boston, USA, 2013)

56. Hughes, R. J., Nordholt, J. E., Derkacs, D. \& Peterson, C. G. Practical free-space quantum key distribution over $10 \mathrm{~km}$ in daylight and at night. New J. Phys. 4, 43-43, https://doi.org/10.1088/1367-2630/4/1/343 (2002).

57. Fang, X. et al. Coupled orbital angular momentum conversions in a quasi-periodically poled litao3 crystal. Opt. Lett. 41, 1169-1172, https://doi.org/10.1364/OL.41.001169 (2016).

58. Sephton, B., Dudley, A. \& Forbes, A. Revealing the radial modes in vortex beams. Appl. Opt. 55, 7830, https://doi.org/10.1364/ AO.55.007830 (2016)

59. Gisler, T. et al. Mode-selective dynamic light scattering: theory versus experimental realization. Appl. Opt. 34, 3546-3553, https:// doi.org/10.1364/AO.34.003546 (1995).

60. Winzer, P. J. \& Leeb, W. R. Fiber coupling efficiency for random light and its applications to lidar. Opt. Lett. 23, 986, https://doi. org/10.1364/OL.23.000986 (1998).

61. Wu, Y. \& Chiang, K. S. Mode-selective coupling between few-mode fibers and buried channel waveguides. Opt. Express 24, 30108-30123, https://doi.org/10.1364/OE.24.030108 (2016).

62. Blochet, B., Bourdieu, L. \& Gigan, S. Focusing light through dynamical samples using fast continuous wavefront optimization. Opt. Lett. 42, 4994-4997, https://doi.org/10.1364/OL.42.004994 (2017).

63. Toninelli, E. et al. Concepts in quantum state tomography and classical implementation with intense light: a tutorial. Adv. Opt. Photonics 11, 67-134, https://doi.org/10.1364/AOP.11.000067 (2019).

64. Weimer, C. et al. Lidars utilizing vortex laser beams. In Ocean Sensing and Monitoring X, vol. 10631, 106310Q, https://doi. org/10.1117/12.2310042 (International Society for Optics and Photonics, 2018).

65. Mogensen, P. C. \& Glückstad, J. Phase-only optical encryption. Opt. Lett. 25, 566-568, https://doi.org/10.1364/OL.25.000566 (2000).

66. Tyler, G. A. \& Boyd, R. W. Influence of atmospheric turbulence on the propagation of quantum states of light carrying orbital angular momentum. Opt. Lett. 34, 142-144, https://doi.org/10.1364/OL.34.000142 (2009).

\section{Acknowledgements}

This research was supported in part by National Science Foundation (Award No. 1842680).

\section{Author contributions}

Y.-P.H. directed the project. H.Z., and S.K. carried out the experiments and prepared the figures. All authors contributed to the theoretical and experimental research.

\section{Competing interests}

The authors declare no competing interests. 
Additional information

Correspondence and requests for materials should be addressed to Y.-P.H.

Reprints and permissions information is available at www.nature.com/reprints.

Publisher's note Springer Nature remains neutral with regard to jurisdictional claims in published maps and institutional affiliations.

(c) (i) Open Access This article is licensed under a Creative Commons Attribution 4.0 International License, which permits use, sharing, adaptation, distribution and reproduction in any medium or format, as long as you give appropriate credit to the original author(s) and the source, provide a link to the Creative Commons license, and indicate if changes were made. The images or other third party material in this article are included in the article's Creative Commons license, unless indicated otherwise in a credit line to the material. If material is not included in the article's Creative Commons license and your intended use is not permitted by statutory regulation or exceeds the permitted use, you will need to obtain permission directly from the copyright holder. To view a copy of this license, visit http://creativecommons.org/licenses/by/4.0/.

(C) The Author(s) 2019 